\title{
Quantification of Tooth Wear by Selected Desensitizing Polishing Pastes Using White Light Profilometry
}

\author{
Hussain HF, Hill RG and Gillam DG* \\ Institute of Dentistry, Barts and the London School of Medicine and Dentistry, Queen Mary University London, London E1 2AD, UK
}

${ }^{\star}$ Corresponding author: David G Gillam, Centre for Oral Bioengineering, Institute of Dentistry, Barts and the London School of Medicine and Dentistry, Queen Mary University London, London E1 2AD, UK; E-mail: d.g.gillam@qmul.ac.uk

Received: November 17, 2020; Accepted: November 26, 2020; Published: November 30, 2020

\begin{abstract}
Objectives: To analyse tooth wear using white light non-contact profilometry following the polishing of the tooth surface with selected polishing pastes.

Methods: Three polishing pastes containing a range of particles sizes and different coarseness (extra-fine, medium, course) were compared with commercially available prophylaxis pastes (Nupro with Novamin ${ }^{\circledR}$ and Nupro with Fluoride) as controls. Particle size distribution was analysed using a using particle size analyser and quantified using Masterizer software. Teeth were in $70 \%$ ethanol prior to evaluation. 25 extracted human premolar teeth were distributed in five groups $(n=5)$, and the teeth were mounted in a silicone putty matrix leaving an exposed buccal surface. White light profilometry with Proscan 2000 software was used to scan each tooth surface before and after polishing. Scantron ProForm software was used to superimpose images and measure surface loss and analyse the difference between the two surfaces-scans by the Proscan 2000 software.
\end{abstract}

Results: Particle size analysis indicated that all samples consisted of a wide distribution of particles' sizes (DX 10, 50, and 90). The course polishing paste had the largest DX 90 whereas Nupro with Fluoride had the lowest DX 90 . The extra-fine pumice had the lowest DX 90, although this paste had larger values for DX 10 and DX 50 compared to the medium paste. The volume tooth loss analysis demonstrated that the course pumice had the most tooth surface loss compared to the extra-fine pumice which had the least amount of tooth surface loss. The average volume loss per group was $0.808,0.022$, 0.014, 0.022, 0.026 (course, medium, extra-fine, Nupro with Fluoride, and Nupro with Novamin ${ }^{\circledR}$ ) respectively.

Conclusions: The results indicated that the larger the DX 90 within the paste, the more tooth surface loss occurred due to the abrasivity of the paste. There was however minimal or no significant difference in the amount of tooth loss between the control polishing pastes.

Keywords: Prophylaxis polishing pastes, Abrasion, White light profilometry, Particle size analysis

\section{Introduction}

Dental materials are frequently used in polishing procedures during periodontal procedures in daily dental practice and the abrasives in these materials may subsequently have an impact on tooth surface loss and wear. Several factors are indicated in the aetiology of tooth wear with or without Dentine Hypersensitivity (DH) such as erosion, attrition and, abrasion. Furthermore, different materials other than a tooth can cause tooth contact when it contacts a tooth (so-called two-body or threebody contact [Tribology]) [1]. The term wear is, therefore, a better descriptive term to define the loss of tooth structure [2]. Tooth wear can be defined as the net loss of tooth structure when it is under function [1]. Previous studies have reported a growing interest in quantifying tooth structure loss which is called 'wear quantification' both in vivo and in vitro in three dimensions. Volume and mean height are the most clinically relevant parameters that can be used to analyse tooth loss [3]. It is essential to have a systematic, reliable and, repeatable data using a wear quantification method. The method itself is time consuming, which requires an experienced operator to apply the different software packages that are available commercially for wear quantification [4]. It is, however, a useful method to compare and evaluate the effect of different new materials, which may cause tooth wear in vitro. An accurate surface topographic representation of a tooth both pre- and post-wear testing is essential for any in vitro wear qualification to be valid. There are three main types of sensors that are used for scanning and subsequently quantifying the wear namely: 1) contact sensors [5], 2) non-contact sensors [6] and 3) white light [7] which are all suitable for systematic studies [4]. Investigators have previously utilised white light non-contact profilometric techniques as a quantifiable measure of tooth loss/abrasive wear and/or erosion [7-9]. White light profilometry uses effective sensors to measure the distance in which they can split the white light beam into its constituent wavelength [10]. Each wavelength matches to its corresponding distance which creates its monochromatic image point. Therefore, the image reflects the surface topography of a scanned specimen which it can provide a quantitative measure of shape, texture, microtopography, microform and roughness [10].

\footnotetext{
Aim

The aim of this in vitro study was to analyse tooth wear on extracted human teeth using contactless white light profilometry following professional polishing with selected polishing pastes with different types of pumice used in the polishing of teeth during periodontal procedures.
} 


\section{Material and Method}

This exploratory study was based on two procedures. The first part described in this paper was to quantify tooth wear using a white light profilometry following polishing of the teeth to choose the ideal abrasivity of the pumice that would be incorporated into future prophy-paste formulations. The second part of the study was the evaluation of selected pastes to determine their effectiveness in tubular occlusion and this will be reported in a subsequent paper.

\section{Particle Size Analysis}

The same weight $(50 \mathrm{mg}$ ) of the polishing prophylaxis paste samples were dissolved separately in $50 \mathrm{~mL}$ deionised water. Once the solid particles were dispersed, the diluted solution was transferred into a system that uses the MASTERSIZER 3000E (MALVERN software) to initiate the measurements and analysing the particle sizes through the laser diffraction method using a dispersion of particles in a liquid, wet, Hydro $E V$, deionised water with a 1.33 refractive index. The Mastersizer $\mathrm{E}$ used is designed to obtain values for a wide particle size range of 0.1 to $3500 \mu \mathrm{m}$. The setting of the software was pre-set manually to a duration of 15 seconds background measurement(s) and 10 seconds sample measurements. The diluted solution was added in small quantities until the obstruction range of 5-20\% was achieved. A speed of $2000 \mathrm{rpm}$ for the hydro pump speed was used for all tested samples. Four different measurements for each sample were automatically reported, analysed, and averaged by the software. The median for different volume distributions DX 10, 50, and 90 were recorded and the data was subsequently exported into an Excel file for analysis.

\section{Preparation of Materials}

A total of 30 extracted, caries free human premolars were collected from the walk-in dental polyclinics from Kuwait in 2017 after obtaining verbal consent from patients for the use of their teeth in research. The teeth were stored in a small container of Listerine mouthwash (Johnson and Johnson, UK) and brought to the UK by HFH under QMUL guidelines UK. The teeth were transferred and stored in a $70 \%$ Ethanol solution in a specimen container at room temperature within the Department of Physical Sciences Unit at Mile End, London in accordance with HTA regulations. The extracted premolars were distributed into five groups $(n=5)$ and teeth were mounted in a silicon putty matrix (Zetaplus plus mixed with an indurent gel (Zhermack SpA, Italy), leaving an exposed buccal surface to evaluate. The groups were numbered from 1 to 5 and they were stored in the $70 \%$ Ethanol solution at room temperature within the Department.

Prior to scanning the samples were prepared by placing three divots using a $1 / 2$ round bur at high speed on the flattest buccal (facial) surface of each tooth. Three polishing pumices with a range of particle sizes and different coarseness of pumice (extra-fine, medium, course) (Kemdent Works, Swindon UK) were compared to commercially available prophylaxis pastes namely, Nupro with Novamin ${ }^{\circ}$, Nupro with Fluoride (Dentsply International, USA) (Controls). A battery-operated dental polisher portable handpiece (Dentitex model number TP-01; 8000 rpm motor), was used as a polishing carrier device instead of a slow speed handpiece for practical purposes. Its cup has the same size as a dental office polishing cup. To avoid any contamination of the materials, each cup was dedicated for a specific prophylaxis paste. Three different pumice powders (course, medium and extra-fine) (Kemdent; Swindon, UK) were characterized in terms of their particle size distribution (Masterizer software). The exact weight measure of the samples was dissolved separately in $50 \mathrm{mg}$ deionised water. The diluted solution was transferred to initiate the measurements and analysing the particle sizes through a laser diffraction method using a dispersion of particles in a liquid, Hydro EV, deionised water with 1.33 refractive index (Mastersizer 3000E from Malvern software).

\section{Quantification of Tooth Surface Loss}

\section{White Light Profilometry (WLP)}

Two software programmes were used for analysing the tooth surface loss; namely: a Proscan 2000 and a Scantron ProForm. The Proscan 2000 software is designed for shape analysis, object digitisation and accurate surface analysis. The Scantron ProForm software is designed for analysing the differences between two surfaces-scans made by the Proscan 2000 software accuracy.

Three divots on the buccal surface of every tooth were placed to define reference points and the surfaces scanned. The pumice was used with water only, and the tooth was polished for two minutes using the portable polishing handpiece (Dentitex). The tooth was gently rinsed with water until all pumice particles were no longer observed on the tooth surface. A second scan was undertaken using white light profilometry. The two scans were then superimposed in a different software Scantron ProForm to measure any surface volume loss and analyse the difference between the two surfaces-scans. An area of $0.4 \mathrm{x}$ $0.4 \mu \mathrm{m}^{2}$ was randomly selected between the three divots as a standard dimension for all samples (Figure 1).

\section{Results and Discussion}

The particle size analysis showed that all samples consisted of a wide distribution of particle sizes (DX 10, 50, and 90). Table 1 and Figure 2 show the particle size distribution for each sample. The course pumice sample had the largest amount of DX 90 particle size whereas Nupro with Fluoride had the smallest DX 90. The extra-fine pumice sample had the smallest DX 90 for the pumice powders but had larger values for DX 10 and DX 50 than the medium pumice sample.

The tooth surface loss volume was analysed using white light non-contact profilometry following the polishing of the tooth surface with the selected polishing pastes. The results demonstrated that the course pumice had the most tooth surface loss compared to the extrafine pumice which had the least amount of tooth surface loss. The average volume loss per group was $0.808,0.022,0.014,0.022,0.026$ $\mathrm{mm}^{3}$ (course, medium, extra-fine, Nupro with Fluoride, and Nupro with Novamin ${ }^{\oplus}$ ) respectively (Table 2 and Figure 3 ). The t-test between the Medium vs. Extra-Fine samples was 0.0098 which indicated a significant difference in surface loss. Based on this result an extra-fine pumice was recommended to be incorporated in the prophy-paste formulation in subsequent studies. The results indicated that the larger 

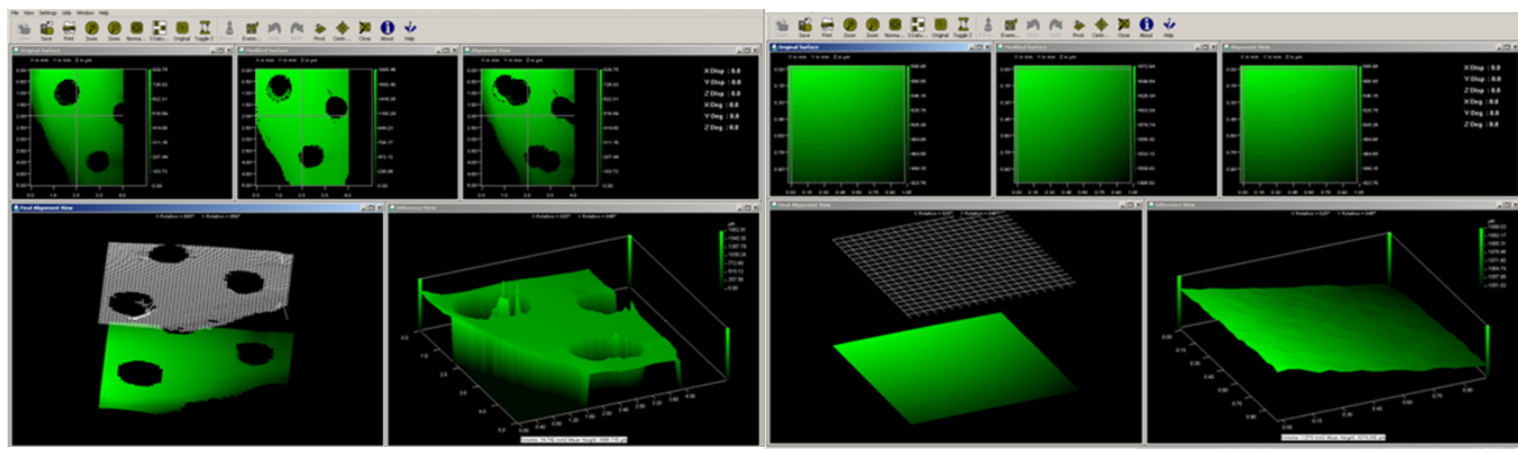

Figure 1: shows a) superimposing of pre-treated and post-treated tooth surface using course pumice. b) Random selection of area $0.4 \mathrm{x} 0.4 \mu \mathrm{m}^{2}$ between the three created divots on the tooth.

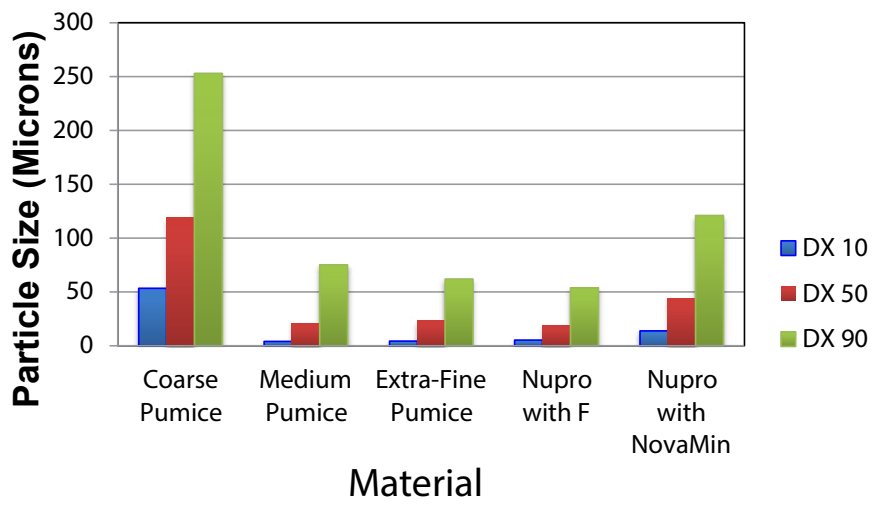

Figure 2: Particle Size Distribution DX 10, 50, and 90 particle sizes of the five materials.

Table 1: Distribution of DX 10, 50, and $90 \mu \mathrm{m}$ particle sizes of the five groups.

\begin{tabular}{|l|c|c|c|}
\hline & DX 10 $(\boldsymbol{\mu m})$ & DX 50 $(\boldsymbol{\mu m})$ & DX 90 $(\boldsymbol{\mu m})$ \\
\hline Course Pumice & 53.4 & 119 & 253 \\
\hline Medium Pumice & 4.04 & 21.0 & 75.1 \\
\hline Extra-Fine Pumice & 4.33 & 23.7 & 62.0 \\
\hline Nupro with F & 5.27 & 19.1 & 53.8 \\
\hline Nupro with NovaMin & 13.8 & 44.4 & 121 \\
\hline
\end{tabular}

Table 2: The average of tooth surface loss in $\left(\mathrm{mm}^{3}\right)$ for the different materials analysed where $\mathrm{T}$ is the tooth sample that was used.

\begin{tabular}{|l|c|c|c|c|c|}
\hline Sample/Material & Course & Medium & Extra-Fine & $\begin{array}{c}\text { Nupro with } \\
\text { Fluoride }\end{array}$ & $\begin{array}{c}\text { Nupro with } \\
\text { Novamin }\end{array}$ \\
\hline T1 & 1.074 & 0.019 & 0.013 & 0.0303 & 0.021 \\
\hline T2 & 0.708 & 0.021 & 0.009 & 0.0196 & 0.017 \\
\hline T3 & 0.633 & 0.017 & 0.015 & 0.008 & 0.026 \\
\hline T4 & 0.877 & 0.029 & 0.017 & 0.0236 & 0.039 \\
\hline T5 & 0.749 & 0.025 & 0.018 & 0.0294 & 0.028 \\
\hline Average & 0.8082 & 0.0222 & 0.0144 & 0.0222 & 0.0262 \\
\hline Standard Deviation & 0.1729 & 0.0048 & 0.0036 & 0.0091 & 0.0083 \\
\hline
\end{tabular}

the DX 90 value of the paste, the more tooth surface loss occurred due to the abrasivity of the paste. Thus, it seems that the coarse particles in the particle size distribution close to DX 90 dominate the tooth loss. There were no significant differences in the amount of tooth loss between the two control samples.

Table 2 shows the average of tooth surface loss in $\left(\mathrm{mm}^{3}\right)$ for the different materials analysed where $\mathrm{T}$ is the tooth sample that was used.

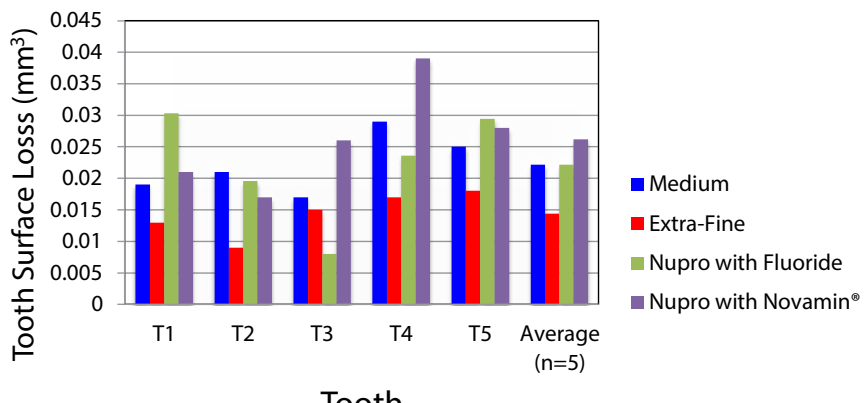

Tooth

Figure 3: The average of tooth surface loss $\left(\mathrm{mm}^{3}\right)$ between the selected prophy-pastes after removing the course particle sample: $\mathrm{T}$ is the tooth sample that was used.

\section{Conclusion}

The results from this exploratory study on the effect of the particle size distribution on tooth surface loss indicated that the larger the DX 90 particle size of the pumice samples, the more tooth surface loss and wear. The extra-fine pumice sample should be incorporated into a prophylaxis paste to reduce any potential tooth surface loss.

\section{Reference}

1. Addy M (2000) Dentine hypersensitivity: Definition, prevalence, distribution and etiology. In: Addy M, Embery G, Edgar WM, Orchardson R, editors. Tooth wear and sensitivity: Clinical advances in restorative dentistry. London: Martin Dunitz 2000: 239-248.

2. Smith RG (1997) Gingival recession. Reappraisal of an enigmatic condition and a new index for monitoring. J Clin Periodontol 24: 201-205. [crossref]

3. Pintado MR, Anderson GC, DeLong R, Douglas WH (1997) Variation in tooth wear in young adults over a two-year period. J Prosthet Dent 77: 313-320. [crossref]

4. Heintze SD, Cavalleri A, Forjanic M, Zellweger G, Rousson V (2006) A comparison of three different methods for the quantification of the in vitro wear of dental materials. Dent Mater 22: 1051-1062. [crossref]

5. Magne P Oh WS, Pintado MR, DeLong R (1999) Wear of enamel and veneering ceramics after laboratory and chairside finishing procedures. J Prosthet Dent 82: 669679. [crossref]

6. Mehl A, Gloger W, Kunzelmann KH, Hickel R (1997) A new optical 3-D device for the detection of wear. J Dent Res 76: 1799-1807. [crossref]

7. Vieira A, Overweg E, Ruben JL, Huysmans MC (2006) Toothbrush abrasion, simulated tongue friction and attrition of eroded bovine enamel in vitro. J Dent 34 : 336-342. [crossref]

8. Hara AT, Zero DT (2008) Analysis of the erosive potential of calcium-containing acidic beverages. Eur J Oral Sci 116: 60-65. [crossref] 
Gillam DG (2020) Quantification of Tooth Wear by Selected Desensitizing Polishing Pastes Using White Light Profilometry

9. Theocharopoulos A, Zou L, Hill R, Cattell MJ (2010) Wear quantification of human enamel and dental glass-ceramics using white light profilometry. Wear 269: 930993.
10. Litwin D, Galas J, Blocki N (2006) Variable wavelength profilometry, in: Proceedings of the Symposium on Photonics Technologies for 7th Framework Program (Wroclaw, 2006). 476-479.

\section{Citation:}

Hussain HF, Hill RG, Gillam DG (2020) Quantification of Tooth Wear by Selected Desensitizing Polishing Pastes Using White Light Profilometry. J Dent Maxillofacial Res Volume 3(4): 1-4. 